\title{
Detection of Periodic Signals in Noise Based on Higher-Order Statistics Joined to Convolution Process and Spectral Analysis
}

\author{
Miguel Enrique Iglesias Martínez and Fidel Ernesto Hernández Montero \\ Universidad de Pinar del Río Hermanos Saíz Montes de Oca, \\ Departamento de Telecomunicaciones, Calle Martí Final No. 270, \\ esquina 27 de Noviembre, Pinar del Río, Cuba, C.P 20100 \\ migueliglesias2010@gmail.com, fidel@tele.upr.edu.cu
}

\begin{abstract}
This paper refers to the application of higher-order statistical signal processing techniques (cumulant calculation) on Gaussian noise cancellation. The performed procedure, joined to a convolution process and Fast Fourier Transform (FFT) application, results in the complete estimation (i.e., amplitude, frequency and phase recovery) of any corrupted periodic signal. Whereas tone frequency estimation is performed by 4th-order cumulant calculation, phase recovery is achieved by the convolution of the cumulant calculation and the corrupted signal. At last, the original signal amplitude is recovered by means of modification of the resulting amplitude spectrum. In this paper, higher-order statistics foundations are presented and the validation of the proposed algorithm is revealed in both theoretical and practical sense. Obtained results are highly satisfactory.
\end{abstract}

Keywords: Higher-Order Statistics, Noise Cancellation, Convolution, Fourier Transform.

\section{Introduction}

Methods for noise cancellation using higher-order statistics, in particular those based on cumulants, have demonstrated to be very effective [1]. This is mainly due to the properties that state, from one hand, that higher-order cumulants of a normal distribution random signal is 0 and, on the other hand, that cumulant of the sum of signals is equal to the sum of the cumulants of each signal, then higher-order cumulant of a signal corrupted by normal distribution random signal (noise) is equal to the higher-order cumulant of the signal without noise.

Several works have been approached to the harmonics retrieval problem [2], [3] and [4]. However all of these papers only reach to recover harmonic amplitude and frequency; phase cannot be retrieved. Other works, as in [5] and [6], use higher-order statistics (fourth-order cumulant) for adaptive estimation of amplitude and frequency of harmonics in real zero-mean random signals. Some methods, developed for phase 
estimation, are based on higher-order spectra [7] [8] and Bayes Theorem [9]. However, tools proposed in these works exhibit a high computational complexity (higher-order spectral parameters are results of multidimensional functions calculation) that makes them no suitable for practical use and cannot be directly or simply applied on the problem of estimation of amplitude, frequency and phase of a periodic signal in noise.

Methods for phase estimation using Polyspectrum Slice [10] have also been reported but these ones are just approached to phase recovery in linear time-invariant systems, thus they are out of the scope of this work. In order to obtain a complete algorithm for periodic signals estimation corrupted by noise, a new algorithm is proposed in this work.

\section{Removing Noise from Periodic Signal through Higher-Order Statistics}

For real value signals, in the problem that concerns removing noise from harmonic signal, observed data is described as follows:

$$
y(t)=\sum_{k=1}^{N} A_{k} \cos \left(w_{k} t+\phi_{k}\right)+w(t)=x(t)+w(t)
$$

where $x(t)$ is the useful signal (signal to be detected) and $w(t)$ is additive zero mean Gaussian noise. Besides, $A_{k}, f_{k}$ and $\phi_{k}$ are the amplitude, frequency and phase, respectively, of the signal. Since higher-order cumulants of a zero mean Gaussian noise is equal to zero, the estimation of cumulants for noise cancellation can be made starting from the third order, but from [11] all third-order cumulants of complex harmonic are always zero. Consequently this research continues with the use of fourth-order cumulant.

\subsection{Fourth-Order Cumulant Calculation}

For a zero-mean stationary random process $z(t)$, and for $k=3,4$, the kth order cumulant of $z(t)$ can be defined in term of its joint moments as [12]:

$$
C_{k}^{z}\left(\tau_{1}, \tau_{2}, \ldots, \tau_{k-1}\right)=E\left\{z\left(\tau_{1}\right) \ldots z\left(\tau_{k-1}\right)\right\}-E\left\{g\left(\tau_{1}\right) \ldots g\left(\tau_{k-1}\right)\right\}
$$

Where $g(t)$ is a Gaussian random process with the same second-order statistic as $z(t)$. If $z(t)$ is Gaussian, the cumulants are all zero. Then, for zero-mean real random variables the fourth-order cumulant can be calculated in agreement to [12] as follows:

$$
\begin{gathered}
C_{4}^{z}\left(\tau_{1}, \tau_{2}, \tau_{3}\right)=E\left\{z(t) \cdot z\left(t+\tau_{1}\right) \cdot z\left(t+\tau_{2}\right) \cdot z\left(t+\tau_{3}\right)\right\} \\
-C_{2}^{z}\left(\tau_{1}\right) \cdot C_{2}^{z}\left(\tau_{2}-\tau_{3}\right)-C_{2}^{z}\left(\tau_{2}\right) \cdot C_{2}^{z}\left(\tau_{3}-\tau_{1}\right)-C_{2}^{z}\left(\tau_{3}\right) \cdot C_{2}^{z}\left(\tau_{1}-\tau_{2}\right)
\end{gathered}
$$


According to the process described in equation (1), since $w(t)$ is a zero-mean Gaussian random signal, $C_{4}^{w}\left(\tau_{1}, \tau_{2}, \tau_{3}\right)=0$. Then, $C_{4}^{y}\left(\tau_{1}, \tau_{2}, \tau_{3}\right)=C_{4}^{x}\left(\tau_{1}, \tau_{2}, \tau_{3}\right)$.

Using equation (3) and working with only the one-dimensional component of the fourth-order cumulant, $C_{4}^{y}\left(\tau_{1}, 0,0\right)$, by setting $\tau_{2}=\tau_{3}=0$, leads to a result similar to that obtained in [11] by setting $\tau_{1}=\tau_{2}=\tau_{3}=\tau$. This one-dimensional component contains original amplitude and frequency of the signal to detect, $x(t)$, although the phase is missed; on the other hand, the noise is entirely removed:

$$
C_{4}^{y}\left(\tau_{1}, 0,0\right)=E\left\{x(t)^{3} \cdot x\left(t+\tau_{1}\right)\right\}-3 \cdot E\left\{x(t) \cdot x\left(t+\tau_{1}\right)\right\} \cdot E\left\{x^{2}(t)\right\}
$$

Then, developing the left term of the equation (4) by substituting $x(t)$ declared in (1):

$$
\begin{aligned}
E\left\{x(t)^{3} \cdot x\left(t+\tau_{1}\right)\right\} & =\int_{-\pi}^{\pi} g(x) \cdot f_{\varphi}(\phi) d \phi \\
& =\sum_{k=1}^{N} \frac{3 A_{k}^{4}}{8} \cos \left(w_{k} \tau_{1}\right)
\end{aligned}
$$

Substituting, in the equation (6), the result obtained in the expression (5):

$$
C_{4}^{y}\left(\tau_{1}, 0,0\right)=\sum_{k=1}^{N} \frac{3 A_{k}^{4}}{8} \cos \left(w_{k} \tau_{1}\right)-\sum_{k=1}^{N} \frac{3 A_{k}^{4}}{4} \cos \left(w_{k} \tau_{1}\right)
$$

obtaining as result (similar to that obtained in [11]):

$$
C_{4}^{y}\left(\tau_{1}, 0,0\right)=\sum_{k=1}^{N}-\frac{3 A_{k}^{4}}{8} \cos \left(w_{k} \tau_{1}\right)
$$

It is clear from equation (7), that the waveform of the original signal is not preserved, which is due to the loss of the phase information of the original signal in the noise cancellation procedure. This is the problem to face in the following section.

\section{Phase Recovery Method}

In order to preserve the phase information of the original signal (deterministic) in $C_{4}^{y}\left(\tau_{1}, 0,0\right)$, a method based on the convolution between corrupted signal, $\mathrm{y}(\mathrm{t})$, and $C_{4}^{y}\left(\tau_{1}, 0,0\right)$ is proposed. In order to theoretically prove the proposed method, let $a(t)$ be a sinusoidal signal corrupted by Gaussian noise, 
$a(t)=\sum_{k=1}^{N} A_{k} \cos \left(w_{k} t+\phi_{k}\right)+w(t)$, and $b(t)$, the one-dimensional 4th-order cumulant of the corrupted signal, $C_{4}^{y}\left(\tau_{1}, 0,0\right)=\sum_{k=1}^{N}-\frac{3 A_{k}^{4}}{8} \cos \left(w_{k} \tau_{1}\right)$, (i.e., an equivalent of the free-noise periodic signal, the phase of which is equal to 0 ). The convolution procedure is developed as follows:

$$
\begin{gathered}
\operatorname{Conv}_{a b}=\lim _{T \rightarrow \infty} \frac{1}{T} \int_{-T / 2}^{T / 2} a(t) \cdot b(-t+\tau) d t \\
=\int_{-T / 2}^{T / 2} \sum_{k=1}^{N}\left[A_{k} \cos \left(w_{k} t+\phi_{k}\right)+w(t)\right]\left[-\frac{3 A_{k}{ }^{4}}{8} \cos \left(-w_{k} t+w_{k} \tau\right)\right] d t \\
=\int_{-T / 2}^{T / 2} \sum_{k=1}^{N}-\frac{3 A_{k}{ }^{5}}{8} \cos \left(w_{k} t+\phi_{k}\right) \cdot \cos \left(-w_{k} t+w_{k} \tau\right) d t \\
+\int_{-T / 2}^{T / 2} \sum_{k=1}^{N}-\frac{3 A_{k}^{4}}{8} \cos \left(-w_{k} t+w_{k} \tau\right) \cdot w(t) d t \\
\operatorname{Conv}_{a b}=\sum_{k=1}^{N}-\frac{3 A_{k}{ }^{5}}{16} \cos \left(w_{k} \tau+\phi_{k}\right)
\end{gathered}
$$

Equation (9) reveals that an equivalent of the original periodic signal, preserving phase information, is achieved.

\section{Spectral Amplitude Estimation}

As it can be seen in (9), the original harmonic amplitudes are affected during the whole process by a non linear factor equivalent to:

$$
A_{i}=A_{o} \sqrt[5]{\frac{16}{3}}
$$

where $A_{i}$ represents the original signal amplitude and $A_{o}$ correspond to the output signal amplitude. This represents a problem because each amplitude of harmonics in $\mathrm{x}(\mathrm{t})$ must be independently corrected by itself. Then, in order to fix the original amplitude of harmonics in the periodic signal, in this paper every individual harmonic component is individually adjusted in the amplitude spectrum. The process involves calculating the FFT of the signal resulting from the convolution process, and then, 
applying expression (10) on every single spectral component. Then, the resulting spectral vector is anti transformed, leading to the original signal with noise removed. This spectral adjustment carries on an important inconvenience since noise brought out by the FFT application is also adjusted (depending on its magnitude, this noise could indeed be amplified). In practice, this inconvenience can be reduced by anti transforming the zero-mean adjusted spectrum instead of the original adjusted spectrum. The method diagram can be observed in figure 1 .

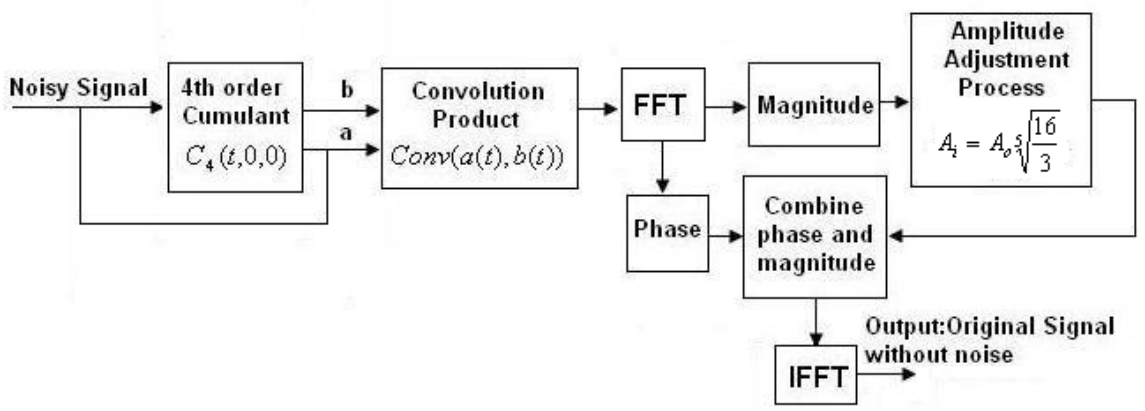

Fig. 1. Block Diagram of the Proposed Algorithm

\section{$5 \quad$ Experimental Results}

First, in order to verify the effectiveness of the proposed algorithm, an experiment using a multitone signal was performed. In this case, the superposition of six tones with different amplitudes $(0.4,0.5,0.6,0.6,0.3,0.1)$, frequencies $(50 \mathrm{~Hz}, 200 \mathrm{~Hz}$, $400 \mathrm{~Hz}, 400 \mathrm{~Hz}, 600 \mathrm{~Hz}$ and $700 \mathrm{~Hz})$ and phases $(\pi / 4 \mathrm{rad}, \pi / 6 \mathrm{rad}, \pi / 3 \mathrm{rad}, \pi / 2 \mathrm{rad}$, $\pi / 12 \mathrm{rad}$ and $\pi \mathrm{rad}$ ), corrupted by zero-mean Gaussian noise, was generated. Figure 2a and $2 \mathrm{~b}$ show the corrupted signal and its spectrum, and the obtained results, applying the proposed algorithm on this signal, are shown in figure 2c.

a)

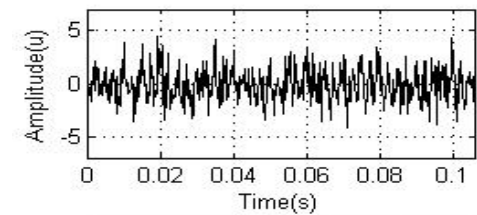

b)

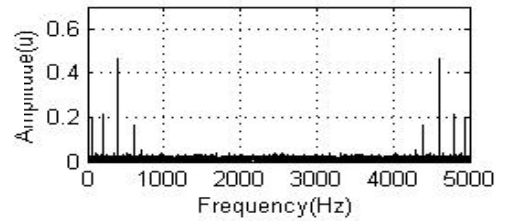

c)

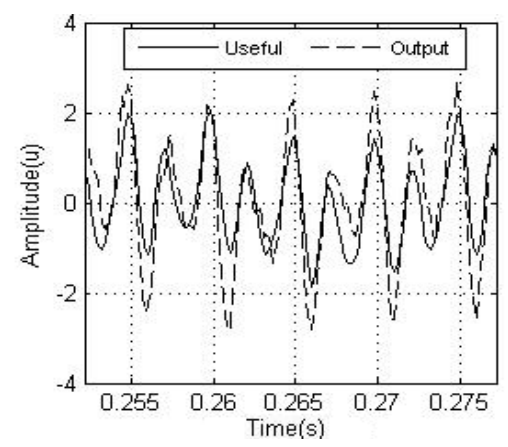

Fig. 2. Sketch of a) Original multitone signal plus noise b) Spectrum of original multitone signal plus noise. c) Comparison between useful signal (uncontaminated signal) and output signal 


\section{- Periodic rectangular and triangular pulses.}

A train of rectangular pulses and a train of triangular pulses were also used during experimentations. The temporal approach of these signals in Fourier series representation can be described through equation (11, rectangular pulses) and (12, triangular pulses) respectively. Figure $3 \mathrm{a}$ and $3 \mathrm{~b}$ show the noisy rectangular pulse and its spectrum, and a comparison between such an output signal and the desired signal is shown in figure $3 \mathrm{c}$. Furthermore, the figure $4 \mathrm{a}$ and $4 \mathrm{~b}$ shows the noisy triangular pulse and its spectrum, and in the figure $4 \mathrm{c}$ it is shown a comparison between the signal at the noise canceller output and the original periodic signal.

$$
\begin{gathered}
x(t)=\frac{4 A}{\pi} \sum_{n=1}^{P} \frac{1}{2 n-1} \sin \left((2 n-1) w_{c} t+\phi\right) \\
x(t)=\frac{8 A}{\pi^{2}} \sum_{n=1}^{P} \frac{(-1)^{((2 n-1)-1) / 2}}{(2 n-1)^{2}} \sin \left((2 n-1) w_{c} t+\phi\right)
\end{gathered}
$$

a)

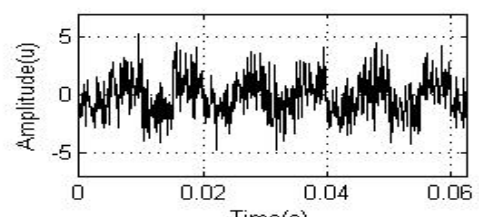

b)

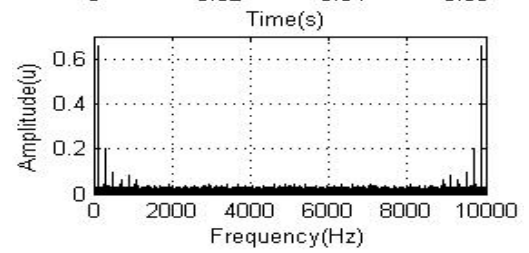

c)

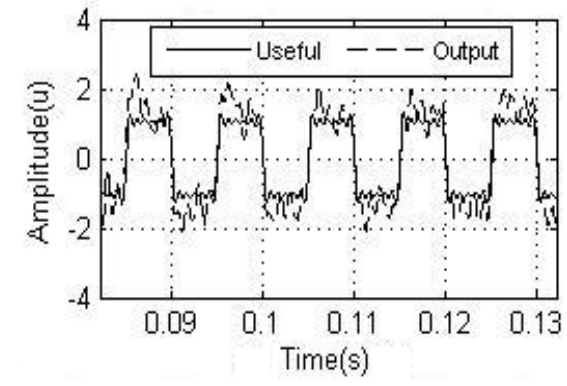

Fig. 3. Sketch of a) Noisy rectangular pulse b) Noisy rectangular pulse spectrum c) Comparison between useful signal (uncontaminated signal) and output signal

a)

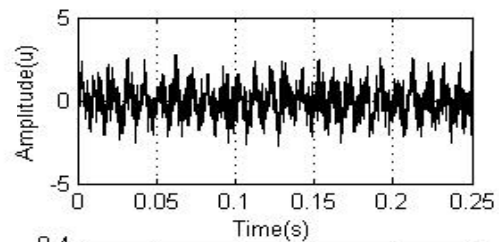

b)

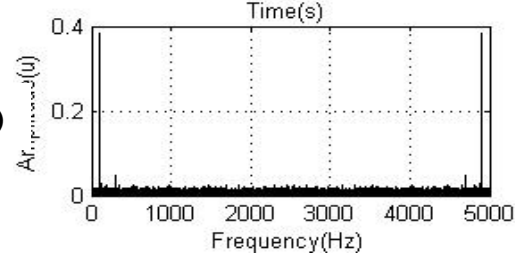

c)

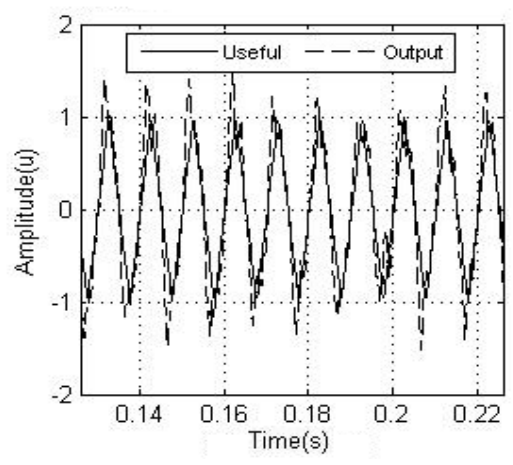

Fig. 4. Sketch of a) Noisy triangular pulse b) Noisy triangular pulse spectrum c) Comparison between useful signal (uncontaminated signal) and output signal 


\section{Working with Real Signals}

This noise cancellation procedure was also applied on real experimental signals. In this case, a signal corresponding to the vibration produced by an unbalanced shaft, as part of an experimental rig, was processed. Since shaft rotates at constant speed, the sensed vibration is periodic by nature. The signal was digitized by a data acquisition system based on an A/D converter, 10 bits resolution, with sampling frequency equals to $20 \mathrm{kHz}$. Gaussian noise was generated in Matlab and added to the sensor signal. The resulting signal was given at the noise canceller input. Figure $5 \mathrm{a}$ and $5 \mathrm{~b}$ show the sensor signal plus noise and its spectrum, and in the Figure $5 \mathrm{c}$ it is shown the output signal and the desired signal. In this experiment, only the correlation index was computed in order to quantify the effectiveness of the noise cancellation procedure; in fact, computation of the SNR at the noise canceller input and output is hard to be accurately achieved. Table 1 summarizes all the results, verifying the effectiveness of the proposed algorithm.

a)



b)

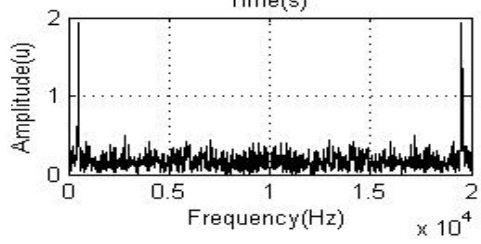

c)

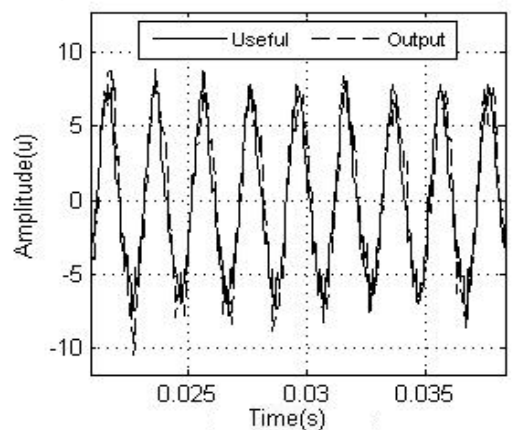

Fig. 5. Sketch of a) Sensor signal plus noise b) Spectrum of sensor signal plus noise c) Comparison between original sensor signal (useful signal) and output signal

Table 1. Results of the proposed noise canceller procedure

\begin{tabular}{|c|c|c|c|c|}
\hline Signal & $\begin{array}{c}\text { Input } \\
\text { SNR(dB) }\end{array}$ & $\begin{array}{c}\text { Output } \\
\text { SNR(dB) }\end{array}$ & $\begin{array}{c}\text { Input } \\
\text { Correlation }\end{array}$ & $\begin{array}{c}\text { Output } \\
\text { Correlation }\end{array}$ \\
\hline Multitone & -3.99 & 2.36 & 0.6332 & 0.8610 \\
\hline Rectangular Pulse & -5.72 & 1.44 & 0.5976 & 0.9575 \\
\hline Triangular Pulse & -6.09 & 1.54 & 0.5883 & 0.9326 \\
\hline Sensor Signal & - & - & 0.5891 & 0.7039 \\
\hline
\end{tabular}

\section{Conclusions}

This research confirmed the advisability of the application of higher-order statistics combined to a convolution process and Fast Fourier Transform, for detection of periodic signals in noise (Gaussian noise). In this work, the use of 4th-order cumulant, 
was proposed and argued. Experimental results performed in Matlab were presented using real and simulate signals, revealing not only the benefits of this application but also the problem it carries on. This problem was clearly defined as the loss of signal phase information because of the noise cancellation procedure. In order to solve this problem, a convolution procedure was proposed. Finally, an amplitude spectral manipulation was performed in order to restore the original amplitude of each spectral component. Results revealed a high effectiveness, given by the significant signal-tonoise rate enhancement achieved, preserving the amplitude, frequency and phase information of the signals to be detected.

\section{References}

1. Howard, R.M.: Principles of Random Signal Analysis and Low Noise Design. John Wiley \& Sons Ltd. (2009)

2. Swami, A., Mendel, J.M.: Cumulant-Based Approach to the Harmonic Retrieval Problem. In: International Conference on Acoustics, Speech, and Signal Processing, ICASSP 1988, April 11-14, vol. 4, pp. 2264-2267 (1988)

3. Swami, A., Mendel, J.M.: Cumulant-Based Approach to Harmonic Retrieval and Related Problems. IEEE Transactions on Signal Processing 39(5), 1099-1109 (1991)

4. Le, T.H., Clediere, J., Serviere, C., Lacoume, J.L.: Noise Reduction in Side Channel Attack Using Fourth-Order Cumulant. IEEE Transactions on Information Forensics and Security 2(4), 710-720 (2007)

5. Zhang, Y., Wang, S.-X.: A Hybrid Approach to Harmonic Retrieval in Non-Gaussian Noise Using Fourth-Order Moment and Autocorrelation. In: Fourth International Conference on Signal Processing, ICSP 1998, vol. 1, pp. 411-414 (1998)

6. Blagouchine, I.V., Moreau, E.: Unbiased Adaptive Estimations of the Fourth-Order Cumulant for Real Random Zero-Mean Signal. IEEE Transactions on Signal Processing 57(9), 3330-3346 (2009)

7. Narasimhan, S.V., Basumallick, N., Chaitanya, R.: Improved Phase Estimation Based on Complete Bispectrum and Modified Group Delay. Signal, Image and Video Processing 2(3), 261-274 (2008)

8. Geng, M., Liang, H., Wang, J.: Research on Methods of Higher-order Statistics for Phase Difference Detection and Frequency Estimation. In: 4th International Congress on Image and Signal Processing, Shanghai, vol. 4, pp. 2189-2193 (2011)

9. Sacchi, M.D., Ulrych, T.J., Walker, C.J.: Interpolation and extrapolation using a highresolution discrete Fourier transform. IEEE Transactions on Signal Processing 46(1), 3138 (1998)

10. Kachenoura, A., Albera, L., Bellanger, J.-J., Senhadji, L.: Non-Minimum Phase Identification Based on Higher Order Spectrum Slices. IEEE Transactions on Signal Processing 56(5), 1821-1829 (2008)

11. Nikias, C.L., Mendel, J.M.: Signal Processsing with Higher-Order Spectra. IEEE Signal Processing Magazine 10(3), 10-37 (1993)

12. Molí, S., Josep M.: Técnicas de Speech Enhancement Considerando Estadísticas de Orden Superior, Tesis Doctoral, Barcelona, Junio (1995),

http: / hal . handle.net/10803/6943 